\title{
Pensononowoor
}

2018, vol. 79, 168-173

http://dx.doi.org/10.12657/denbio.079.015

\author{
Galina Vasilyeva, Valeriya Vavilova, Kirill Ustyantsev, Igor Sukhikh, \\ Alexander Blinov, Sergei Goroshkevich, Victor Sokolov
}

\section{Genetic diversity of Pinus sibirica, $P$. pumila and their natural hybrids based on non-linked nuclear loci}

Received: 13 February 2018; Accepted: 11 May 2018

\begin{abstract}
Frequent discordant phylogenies inferred from different loci, as well as the presence of sufficiently diverged gene variants within a single species isolate are indicative of potentially frequent non-monophyly in the genus Pinus. Interspecies hybridisation and incomplete lineage sorting have been suggested as possible explanations for the observed phylogenetic discrepancies. However, there is no direct evidence to support any of the proposed scenarios for the Eurasian five-needle pines. We used natural hybrids between Pinus sibirica and P. pumila, as well as their parental species, as a model to reproduce the scenario of non-monophyly in the subgenus Strobus. Three non-linked nuclear DNA loci (LEA, AGP6 and 4CL) were applied to detect introgressive alleles and to genetically discriminate the studied species. Comparative sequence analyses revealed two clusters of species-specific alleles for each of the markers, characteristic for either P. sibirica or P. pumila. No hybrid-specific alleles were found. We also found no hybrids with a genotype characteristic of only one of the parental species for all three loci. On average, the hybrids were characterised by an equal ratio of alleles from the $P$. sibirica and $P$. pumila clusters. We reveal that some trees of pure species originating from allopatric locations have non-specific loci that can be a result of genetic exchange between these species in the distant past or incomplete lineage sorting.
\end{abstract}

Keywords: hybridisation, Siberian stone pine, Siberian dwarf pine, genetic variation

Addresses: G. Vasilyeva, S. Goroshkevich, Institute of Monitoring of Climatic and Ecological Systems, Siberian Branch, Russian Academy of Sciences, 10/3, Akademichesky Ave., Tomsk, 634055 Russia, e-mail: galina_biology@mail.ru

V. Vavilova, K. Ustyantsev, I. Sukhikh, A. Blinov, Institute of Cytology and Genetics, Siberian Branch, Russian Academy of Sciences, 10 Prospekt Lavrentyeva, Novosibirsk, 630090 Russia

A. Blinov, V. Sokolov, Institute of Molecular and Cell Biology, Siberian Branch, Russian Academy of Sciences, Novosibirsk, 630090 Russia

V. Sokolov, Vavilov Research Institute of Plant Industry, Russian Academy of Agricultural Sciences, 42-44, B. Morskaya Street, St. Petersburg, 190000 Russia; Department of Natural Sciences, Novosibirsk State University, 2 Pirogova Str., Novosibirsk, 630090 Russia 


\section{Introduction}

Mechanisms of reproductive isolation are weak amongst pine species (Mirov, 1967). Therefore, the frequency of interspecies crosses under controlled pollination is higher than that under natural conditions (Critchfield, 1986; Lu et al., 2007). Five-needle pines (section Quinquefoliae, subgenus Strobus) are not exception to this rule. Siberian stone pine (Pinus sibirica Du Tour) and Siberian dwarf pine (P. pumila (Pall.) Regel) have the largest geographic distributions, with partial overlap among other five-needle pines. Although natural hybridisation between $P$. sibirica and $P$. pumila has been known for some time (Pozdnyakov, 1952), recent studies have begun to examine different aspects such as morphology, flower phenology, seed production, mating system and growth of hybrid seed progeny (Petrova et al., 2007; Goroshkevich et al., 2008; Petrova et al., 2008; Vasilyeva et al., 2010; Vasilyeva \& Goroshkevich, 2012; Vasilyeva, 2014; Vasilyeva, 2017).

$P$. sibirica and P. pumila have different life-forms. Whereas $P$. sibirica is an upright tree, $P$. pumila is prostrate, with natural hybrids exhibiting intermediate morphology and life-form (Goroshkevich et al., 2008). It is important that the hybrids are fertile and crossed with both parental species (Vasilyeva \& Goroshkevich, 2013). The seed efficiency of such hybrids is quite high and could have an impact on the genetic structure of the mixed population (Vasilyeva \& Goroshkevich, 2012; Vasilyeva, 2014). Hence, hybridisation could have significant evolutionary consequences, such as the production of a novel species or deep introgression.

There are many gaps in the phylogeny of the genus Pinus, with non-monophyly, a consequence of ancient hybridisation or incomplete lineage sorting, particularly problematic in phylogenetic tree construction (Syring et al., 2007a,b; Tsutsui et al., 2009). The use of low-copy nuclear genes has been applied successfully in the study of pine phylogeny at low taxonomic levels (Syring et al., 2007a). Such multiple independent markers can provide more accurate data than cytoplasmic DNA regarding the phylogenetic relationships between closely related species (Syring et al., 2005). Several low-copy nuclear loci have been proposed for the phylogeny of Pinus species (Syring et al., 2005; Syring et al., 2007b; Mglinets et al., 2014). The aim of the study was to examine the genetic diversity of $P$. sibirica and $P$. pumila in the sympatric zone and beyond, as well as that of their natural hybrids based on non-linked nuclear loci, LEA (Late Embryogenesis Abundant (LEA)like gene), 4CL (4-coumarate: CoA ligase) and AGP6 (Arabinogalactan-like protein 6).

\section{Methods}

\section{Sample collection}

Plant material (shoots with needles) was collected from the vegetative or seed progeny of species and hybrids of varying geographic origin (Table 1 in Supplementary material, Fig. 1). Both species and the hybrids were identified morphologically. The hybrids were of an intermediate growth habit and were thus identified as presumable F1. All trees were grown at the "Kedr" field station (scientific collection: $507474)$, situated $30 \mathrm{~km}$ south of Tomsk ( $56^{\circ} 13 \mathrm{~N}$ $84^{\circ} 51 \mathrm{E}, 78 \mathrm{~m}$ above sea level) and managed by the Institute of Monitoring of Climatic and Ecological Systems SB RAS.

\section{Amplification, T-vector cloning, and sequencing}

Total DNA was isolated from fresh pine needles using a DNeasy Plant Mini Kit (QIAGEN) as specified

Table 1. Variability of the nuclear loci in P. sibirica, P. pumila and their hybrids

\begin{tabular}{|c|c|c|c|c|c|}
\hline Locus & Species/hybrids & Sequence length (bp) & $\begin{array}{l}\text { Number of sequence } \\
\text { variants }\end{array}$ & $\begin{array}{l}\text { Number of polymorphic } \\
\text { sites/deletions }\end{array}$ & Nucleotide diversity (๘) \\
\hline \multirow[t]{3}{*}{ LEA $^{*}$} & P. sibirica & 160,182 & 2 & $2 / 1$ & 0.00286 \\
\hline & Hybrids & $117,160,182$ & 4 & $8 / 3$ & 0.00436 \\
\hline & P. pumila & $116-117,182$ & 4 & $6 / 2$ & 0.00366 \\
\hline LEA (Total) & & & 6 & 9 & 0.00567 \\
\hline \multirow[t]{3}{*}{$4 C L$} & P. sibirica & 898 & 4 & 7 & 0.00161 \\
\hline & Hybrids & & 8 & 16 & 0.00623 \\
\hline & P. pumila & & 6 & 15 & 0.00568 \\
\hline 4CL (Total) & & & 9 & 16 & 0.00539 \\
\hline \multirow[t]{3}{*}{$A G P 6$} & P. sibirica & 511 & 6 & 19 & 0.01212 \\
\hline & Hybrids & & 13 & 21 & 0.01650 \\
\hline & P. pumila & & 24 & 13 & 0.00978 \\
\hline AGP6 (Total) & & & 24 & 22 & 0.01662 \\
\hline
\end{tabular}

"Nucleotide diversity for the LEA locus is estimated as Insertion/Deletion polymorphism. 


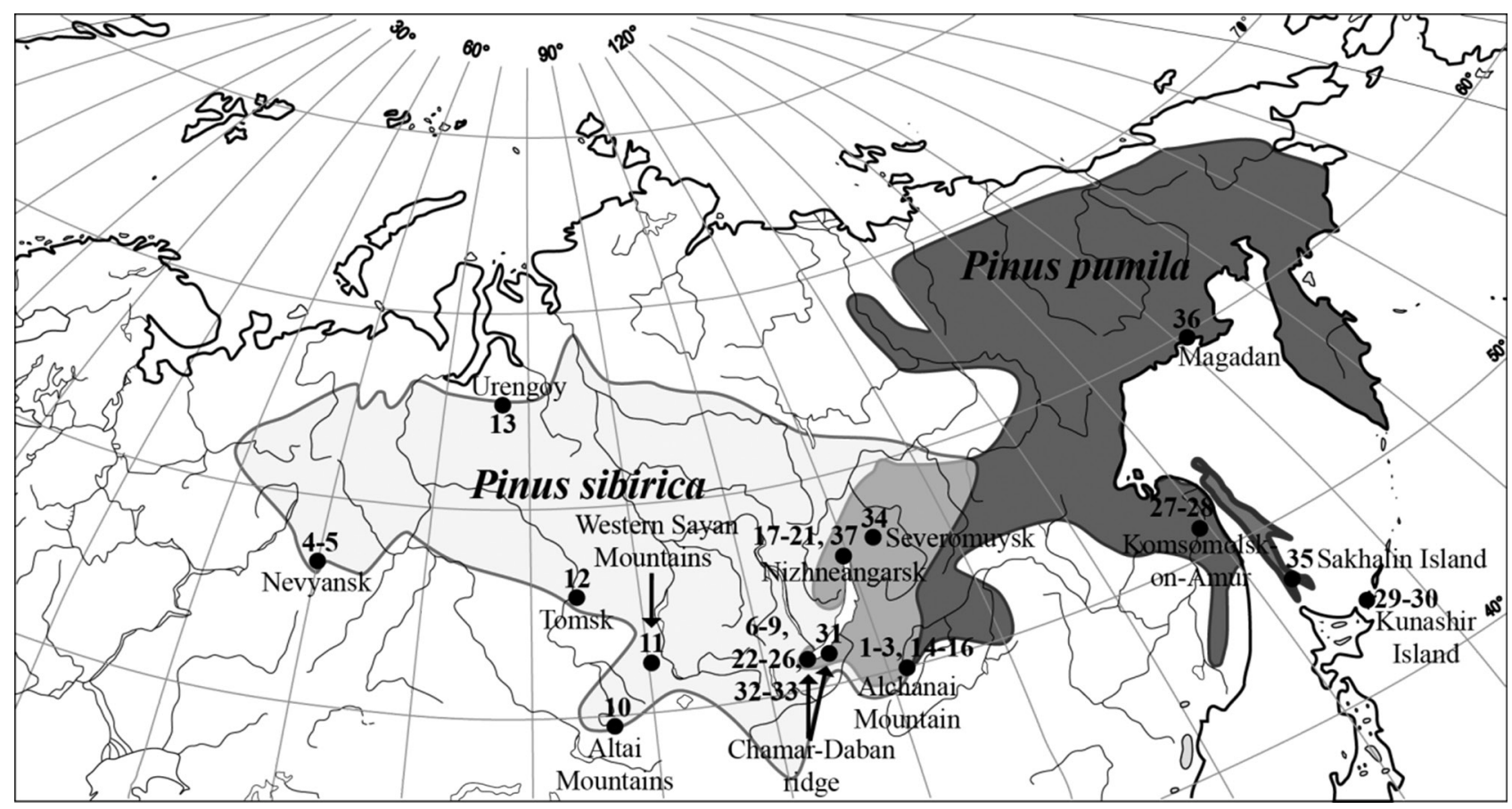

Fig. 1. Geographic distribution of P. sibirica and P. pumila and collection localities (black circles) of the species and hybrids. See Table 1 in Supplementary material for more detailed locality data

in the manufacturer's protocol. The resulting total DNA was amplified via PCR using three pairs of primers, specific to the following partial nuclear gene sequences: $L E A$ (intron fragment), $4 C L$ (the first exon and the following intron) and AGP6 (coding part). PCR conditions and primer sequences for AGP6 and $4 C L$ loci were as in Syring et al. (2005), and for LEA, as in Mglinets et al. (2014). PCR products were separated in $1.5 \%$ agarose gel and purified with a DNA purification kit (BioSilica, http://biosilica.ru/).

The PCR products (final concentration $\sim 0.15 \mu \mathrm{g}$ / $\mu \mathrm{l})$ were ligated into pGEM-T Easy plasmid vector (Promega) in the presence of T4 DNA ligase (Promega) overnight at $16^{\circ} \mathrm{C}$, using a pGEM-T Easy kit (Promega, http://www.promega.com) as specified in the manufacturer's protocol. The ligation products were transformed into E. coli competent cells via standard heat-shock transformation. Obtained white colonies were analysed for the presence of the inserted PCR products using PCR with universal M13 primers.

The inserted PCR products were then sequenced automatically with an ABI PrISM 3100 Avant Genetic Analyzer (Applied Biosystems, USA) using a Big Dye terminator sequencing kit (Applied Biosystems, USA) at the SB RAS Genomics Core Facility (Novosibirsk, Russia, http://sequest.niboch.nsc.ru).

\section{Data analysis}

The number of obtained genetic variants per marker, amount of polymorphic sites and mean number of nucleotide substitutions (or insertions/deletions in the case of $L E A)$ per sequence per site $(\varpi)$ were calculated in DnaSP v. 5.10 (Librado \& Rozas, 2009).

STRUCTURE v.2.3.4 software was used to infer the genetic structure of $P$. sibirica and P. pumila and to identify the species admixture in their hybrids (Hubisz et al., 2009). This particular program employs a Bayesian model-based clustering method; here the best $\mathrm{K}$ (number of clusters) value was identified by running the program for each $\mathrm{K}$ from 1 to 8 with 100000 burn-in cycles followed by 1000000 cycles of data collection (Evanno et al., 2005) based on the use of an admixture model.

Genetic differentiation of $P$. sibirica, $P$. pumila and their hybrids was estimated in Arlequin v. 3.5 (Excoffier \& Lischer, 2010).

\section{Results}

In total, 167 nucleotide sequences were obtained from $L E A, 4 C L$ and AGP6 nuclear loci corresponding to 13 P. sibirica, 11 P. pumila, and 12 P. sibirica $\times$ P. pumila hybrid trees. The obtained nucleotide sequences of $4 C L$ and AGP6 loci were deposited in GenBank (Accession numbers: KT328511-KT328567 and KT447260-KT447315, respectively), while LEA nucleotide sequences were placed in the European Nucleotide Archive (Accession numbers: LN877971LN878024). Six, 9 and 24 sequence variants were identified for $L E A, 4 C L$ and AGP6 loci, respectively. Six unique sequence variants ( 1 and 6 for $4 C L$ and AGP6 loci, respectively) which were not found in the 
Table 2. Genetic diversity and admixture level of $P$. sibirica, $P$. pumila and their hybrids

\begin{tabular}{clccc}
\hline $\begin{array}{c}\text { Grouping } \\
\text { method }\end{array}$ & Species/hybrids & $\begin{array}{c}\text { Average ratio of sibirica-specific } \\
\text { cluster } 1 \pm \text { SD (\%) }\end{array}$ & $\begin{array}{c}\text { Average ratio of pumila-specific } \\
\text { cluster 2 } \pm \text { SD (\%) }\end{array}$ & $\begin{array}{c}\text { Average expected admixture } \\
\text { level } \pm \text { SD (\%) }\end{array}$ \\
\hline Tree morphology & P. sibirica & $90.8 \pm 8.3^{\mathrm{a}}$ & $9.2 \pm 8.3^{\mathrm{a}}$ & $15.4 \pm 10.9^{\mathrm{b}}$ \\
& $\begin{array}{c}\text { P. pumila } \\
\text { Hybrids }\end{array}$ & $4.3 \pm 1.7^{\mathrm{a}}$ & $95.7 \pm 1.7^{\mathrm{a}}$ & $8.2 \pm 2.9^{\mathrm{b}}$ \\
& $\begin{array}{l}\text { Hea of origin } \\
\text { Allopatric }\end{array}$ & $91.7 \pm 28.3^{\mathrm{a}}$ & $61.3 \pm 28.3^{\mathrm{a}}$ & $32.7 \pm 13.1^{\mathrm{c}}$ \\
& $\begin{array}{l}\text { P. sibirica } \\
\text { Sympatric }\end{array}$ & $90.6 \pm 10.7$ & $9.0 \pm 5.3$ & $15.9 \pm 8.5$ \\
& $\begin{array}{l}\text { P. sibirica } \\
\text { Allopatric }\end{array}$ & $4.6 \pm 2.3$ & $9.4 \pm 10.7$ & $15.0 \pm 13.3$ \\
& $\begin{array}{l}\text { P. pumila } \\
\text { Sympatric }\end{array}$ & $3.9 \pm 0.5$ & $95.4 \pm 2.3$ & $8.7 \pm 4.0$ \\
& P. pumila & $96.1 \pm 0.5$ & $7.6 \pm 0.9$ \\
\hline
\end{tabular}

a - significant difference in species-specific allele frequencies found between hybrids and P. sibirica, hybrids and P. pumila, and P. sibirica and P. pumila at the $1 \%$ confidence level (corrected on multiple comparisons by Benjamini-Hochberg procedure) in an unpaired twotailed T-test.

b - significant difference found between P. sibirica and P. pumila average expected admixture level populations at the 5\% confidence level (corrected on multiple comparisons by Benjamini-Hochberg procedure) in an unpaired two-tailed T-test.

${ }^{c}$ - significant difference found between both hybrids and P. sibirica, and hybrids and P. pumila average expected admixture levels at the $1 \%$ confidence level (corrected after multiple comparisons with the Benjamini-Hochberg procedure) in an unpaired two-tailed T-test.

parental species were identified in hybrids (Table 1 in Supplementary material). The diversity of the $L E A$ locus was the largest due to the presence/absence of deletions of 22 and $65 \mathrm{bp}$; in contrast, $4 C L$ and AGP 6 sequences varied only due to single nucleotide polymorphisms (Table 1).

Analysis of genetic structure indicated that the obtained data were better explained by assuming that all observed genetic variation was derived from two clusters $(\mathrm{K}=2)$. These two genetic clusters were species-specific, with $91 \%$ of $P$. sibirica genetic variation associated with the first cluster and $96 \%$ of $P$. pumila genetic diversity with the second cluster, respectively. Genetic variation of hybrids was represented as a mixture of $39 \%$ P. sibirica-specific and $61 \%$ P. pumila-specific genetic clusters. Average expected admixture levels (product of cluster frequencies multiplied by two) comprised $15 \%, 8 \%$ and $33 \%$ for P. sibirica, P. pumila and the hybrids, respectively (Table 2). No differences were recorded between individuals from the allopatric and sympatric zones of the studied species (Fig. 2).

Differentiation between $P$. sibirica and P. pumila $\left(\mathrm{F}_{\text {st }}\right.$ $=0.37)$ is greater than that between $P$. sibirica and
Table 3. Genetic differentiation indices for the studied species and their hybrids

\begin{tabular}{lccc}
\hline & P. sibirica & Hybrids & P. pumila \\
\hline P. sibirica & 1.56000 & 2.20266 & 2.92308 \\
Hybrids & 0.09131 & 2.44308 & 2.59965 \\
P. pumila & 0.37387 & 0.12109 & 2.12121 \\
\hline
\end{tabular}

Grey cells - average number of pairwise differences within $P$. sibiri$c a$, P. pumila and the hybrid groups. Above diagonal - corrected average pairwise differences between $P$. sibirica, $P$. pumila and the hybrids. Below diagonal - pairwise $F_{s t}$ values. All values significant at the $0.1 \%$ confidence level.

hybrids $\left(\mathrm{F}_{\mathrm{st}}=0.09\right)$ and between $P$. pumila and hybrids $\left(\mathrm{F}_{\mathrm{st}}=0.12\right)$, supporting the intermediate position of the hybrids between the parental species (Table 3 ). The hybrids were also characterised by the highest pairwise intrapopulational difference, which is again in accordance with their mixed genetic pattern.

\section{Discussion}

Increased genetic diversity in plant hybrids is a well-known phenomenon (Zalapa et al., 2010 and others). P. sibirica $\times$ P. pumila hybrids are no exception,

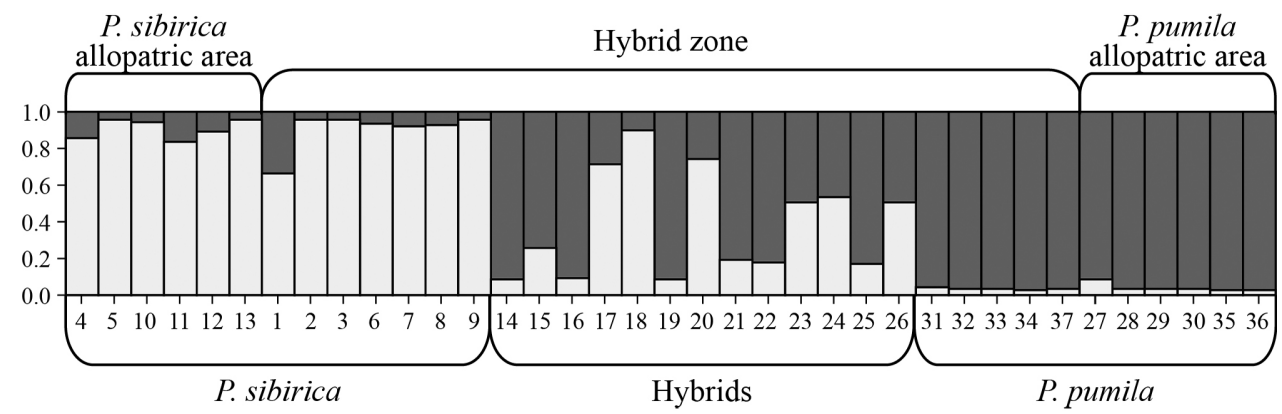

Fig. 2. STRUCTURE v.2.3.4 analysis of $P$. sibirica, $P$. pumila and their hybrids, assuming $\mathrm{K}=2$. Cluster ratios indicated via light and dark grey colours. ID numbers on x-axis as in Table 1 in Supplementary material 
with the results obtained here in good agreement with those of earlier studies (Petrova et al., 2007, 2012; Vasilyeva \& Semerikov, 2014).

Hybrids do not always have an equal ratio of alleles of the parental species. Here we found that whereas the hybrids from the Mount Alchanai region of southern Transbaikalia (sample ID 14-16) were more similar to $P$. pumila, those from northern Pribaikalia (sample ID 17-21) exhibited the reverse pattern and were more similar to P. sibirica. Established patterns of hybrids from distant geographic areas may be due to the duration of hybridisation, which leads to advanced hybrid generations. Hybrids from the northern Pribaikalia possessed pumila-specific mtDNA and sibirica-specific cpDNA (Watano et al., 2006); these hybrids were intermediate relative to their parental species, albeit slightly closer to $P$. sibirica in allozyme profile (Petrova et al., 2010).

The Mount Alchanai isolated population is located at the south-eastern boundary of the P. sibirica's geographic distribution (Bobrinev et al., 2004), and hence at the most south-eastern point of the sympatric zone. Although P. sibirica and P. pumila in this region grow at different altitudes, their distributions overlap at 1500-1600 $\mathrm{m}$ a.s.l., where hybrids are abundant (Petrova et al., 2012). We found that pumila-specific alleles predominated in the hybrids, while allozyme analysis revealed that hybrids were intermediate (presumably F1) and only one hybrid with a prevalence of the $P$. sibirica alleles (presumably backcross) was found (Petrova et al., 2012). Such genetic heterogeneity suggests that interspecies hybridisation in the southern Transbaikalia is of considerable duration and includes at least several generations.

Most intermediate hybrids were found in southern Pribaikalia (Khamar-Daban Ridge, sample ID 2226), but were slightly closer to P. pumila. According to AFLP markers, these hybrids are also intermediate, with the genetic distance to $P$. sibirica slightly less than that to P. pumila (Vasilyeva \& Semerikov, 2014). Thus, the production of advanced generations of hybrids is also possible in the southern Pribaikalia.

We have thus far supposed that all the studied hybrids are F1, based on their intermediate growth habit. However, this intermediate growth habit does not comprise a single growth form but rather a number of possible morphological forms that reflect the contrast in life-form between the parental species. Perhaps, an intermediate growth habit can be retained in more advanced hybrid generations.

Using the non-linked nuclear loci $L E A, 4 C L$ and $A G P 6$, the present study has revealed the genetic admixture in individual $P$. sibirica and P. pumila trees. Previous works have proposed to the use of the LEA gene to confirm the hybrid nature of morphologically intermediate individuals, since $P$. sibirica and $P$. pumila are well differentiated (Mglinets et al., 2014).
However, we found that $P$. sibirica could be heterozygous at the locus in both the sympatric zone and beyond, while $P$. pumila could also be heterozygous beyond the sympatric zone. Therefore, the LEA locus is not a reliable marker for species differentiation and hybrid identification. Furthermore, the same could be said about the $4 C L$ and AGP6 loci, with $P$. sibirica and $P$. pumila potentially heterozygous even outside the current sympatric zone. There are two possible explanations for the obtained results: an ancient genetic exchange and the retention of ancestral polymorphisms.

However, distinguishing introgression and retention of ancestral polymorphisms and incomplete lineage sorting is very difficult. In addition, it may be that both of these factors have contributed to the evolution of the species, as both phenomena are widespread in Pinus species (Syring et al., 2007b; Willyard et al., 2009). Therefore, further research is needed involving both more nuclear loci and increased population numbers representing different regions in the vast ranges of these species.

\section{Conclusion}

We have shown that genetic diversity in $P$. sibirica $\times P$. pumila hybrids is higher than that in the parental species. We found shared genetic polymorphism in $P$. sibirica and $P$. pumila using non-linked nuclear loci. The obtained results suggest possible ancient hybridisation that took place in the distant past or incomplete lineage sorting.

\section{Acknowledgements}

This work was supported by the Russian Academy of Sciences (Program of Basic Research in State Academies, Theme 52.2.6 project No 0369-2016-0001), the Russian Foundation for Basic Research (Project No. 18-04-00833 a), the fundamental scientific research program (project No. 0310-2018-0010), and the Russian Government Budget (project No. 0324-2018-0017).

\section{References}

Bobrinev VP, Pak LN \& Fishchenko VV (2004) Siberian stone pine forests in the eastern Transbaikalia. Publishing House SB RAS, Novosibirsk.

Critchfield WB (1986) Hybridization and classification of the white pines (Pinus section Strobus). Taxon 35: 647-656.

Evanno G, Regnaut S \& Goudet J (2005) Detecting the number of clusters of individuals using the 
software STRUCTURE: a simulation study. Molecular Ecology 14: 2611-2620.

Excoffier L \& Lischer HEL (2010) Arlequin suite ver 3.5: A new series of programs to perform population genetics analyses under Linux and Windows. Molecular Ecology Resources 10: 564-567.

Goroshkevich SN, Popov AG \& Vasilieva GV (2008) Ecological and morphological studies in the hybrid zone between Pinus sibirica and Pinus pumila. Annals of Forest Research 51: 43-52.

Hubisz MJ, Falush D, Stephens M \& Pritchard JK (2009) Inferring weak population structure with the assistance of sample group information. Molecular Ecology Resources 9: 1322-1332.

Librado P \& Rozas J (2009) DnaSP v5: A software for comprehensive analysis of DNA polymorphism data. Bioinformatics 25: 1451-1452.

Lu P, Colombo SJ \& Sinclair RW (2007) Cold hardiness of interspecific hybrids between Pinusstrobus and P. wallichiana measured by post-freezing needle electrolyte leakage. Tree Physiology 27: 243-250.

Mglinets AV, Sokolov VA, Petrova EA \& Goroshkevich SN (2014) Comparative sequence analysis of the LEA gene fragment in Pinus sibirica Du tour and Pinus pumila (Pallas) Regel. Russian Journal of Genetics 50: 149-152.

Mirov NT (1967) The genus Pinus. Ronald Publ., New York.

Petrova EA, Bender OG, Goroshkevich SN, Belokon YS, Belokon MM \& Politov DV (2010) Allozyme variation and needle structure of natural hybrids between Siberian Stone pine and Siberian Dwarf pine. Khvoinye Borealnoi Zony 27: 154-159.

Petrova EA, Goroshkevich SN, Belokon MM, Belokon YS \& Politov DV (2008) Population genetic structure and mating system in the hybrid zone between Pinus sibirica Du Tour and P. pumila (Pall.) Regel at the Eastern Baikal Lake shore. Annals of Forest Research 51: 19-30.

Petrova EA, Goroshkevich SN, Belokon MM, Belokon YS \& Politov DV (2012) Natural hybridization between Siberian stone pine (Pinus sibirica Du Tour) and dwarf Siberian pine (Pinus pumila (Pall.) Regel) at the south-eastern Baikal Lake shore. Khvoinye Borealnoi Zony 30: 152-156.

Petrova EA, Goroshkevich SN, Politov DV, Belokon MM, Popov AG \& Vasilyeva GV (2007) Seed production and genetic structure of populations in natural hybrid zone of Siberian stone pine and Siberian dwarf pine. Khvoynyye borealnoy zony 24: 329-335.

Pozdnyakov LK (1952) Tree form of Siberian dwarf pine. Botanicheskij Journal 37: 688-691.
Syring J, del Castillo RF, Cronn R \& Liston A (2007a) Multiple nuclear loci reveal the distinctiveness of the threatened, neotropical Pinus chiapensis. Systematic Botany 32: 703-717.

Syring J, Farrell K, Businský R, Cronn R \& Liston A (2007b) Widespread genealogical nonmonophyly in species of Pinus subgenus Strobus. Systematic Biology 56: 163-181.

Syring J, Willyard A, Cronn R, \& Liston A (2005) Evolutionary relationships among Pinus (Pinaceae) subsections inferred from multiple low-copy nuclear loci. American Journal of Botany 92: 2086-2100.

Tsutsui K, Suwa A, Sawada K, Kato T, Ohsawa TA \& Watano Y (2009) Incongruence among mitochondrial, chloroplast and nuclear gene trees in Pinus subgenus Strobus (Pinaceae). Journal of Plant Research 122: 509-521.

Vasilyeva GV (2014) Seed efficiency of hybrids between Siberian stone pine and Siberian dwarf pine from northern slope of Khamar-Daban ridge. Vestnik MGUL-Lesnoy vestnik - Forestry Bulletin 1: 85-89.

Vasilyeva GV (2017) Growth and morphogenesis of Siberian stone pine, Siberian dwarf pine and their hybrids: comparative analysis of seed progeny ex situ. Tomsk State University Journal of Biology 38: 28-44.

Vasilyeva GV \& Goroshkevich SN (2012) Seed production and growth of the progeny of hybrids between Siberian stone pine and Siberian dwarf pine in comparison with parental species. Khvoynyye Borealnoy Zony 30: 28-32.

Vasilyeva GV \& Goroshkevich SN (2013) Crossability of Pinus sibirica and P. pumila with their hybrids. Silvae Genetica 62: 61-68.

Vasilyeva GV \& Semerikov VL (2014) Application of amplified fragment length polymorphisms markers to study the hybridization between Pinus sibirica and P. pumila. Annals of Forest Research 57: 175-180.

Vasilyeva GV, Zhuk EA \& Popov AG (2010) Flowering phenology of the Siberian stone pine (Pinus sibirica Du Tour), Japanese stone pine (Pinus pumila (Pall.) Regel.) and their hybrids. Tomsk State University Journal of Biology 1: 61-67.

Watano Y, Futamura T, Petrova E \& Goroshkevich S (2006) Unidirectional hybridization between Pinus pumila and P. sibirica in Lake Baikal region. Journal of Plant Research 119 (Supplement): 55.

Willyard A, Cronn R \& Liston A (2009) Reticulate evolution and incomplete lineage sorting among the ponderosa pines. Molecular Phylogenetics and Evolution 52: 498-511. 